\title{
Molecular cloning, characterization and differential expression of a Sporothrix schenckii STE20-like protein kinase SsSte20
}

\author{
ZHENYING ZHANG $^{1^{*}}$, BINBIN HOU ${ }^{1 *}$, FANGLIANG ZHENG $^{2}$, \\ XIAOHONG YU ${ }^{1}$ and XIAOMING LIU ${ }^{1}$ \\ ${ }^{1}$ Department of Dermatology, The First Affiliated Hospital of Dalian Medical University, Dalian, \\ Liaoning 116011; ${ }^{2}$ Key Laboratory of Animal Resource and Epidemic Disease Prevention, \\ Life Science School of Liaoning University, Shenyang, Liaoning 110036, P.R. China
}

Received January 8, 2013; Accepted March 22, 2013

DOI: 10.3892/ijmm.2013.1352

\begin{abstract}
Dimorphic switching requires fungal cells to undergo changes in polarized growth in response to environmental stimuli. The Ste20-related kinases are involved in signaling through mitogen-activated protein kinase pathways and in morphogenesis through the regulation of cytokinesis and actin-dependent polarized growth. In this report, we isolated and characterized an Ste20 homologue gene, designated SsSte20, from yeast-form Sporothrix schenckii (S. schenckii). The full length SsSte20 cDNA sequence is 2846 bp in size, and contains an open reading frame of 2505 bp encoding 835 amino acids. The predicted molecular mass of SsSte20 is $91.31 \mathrm{kDa}$ with an estimated theoretical isoelectric point of 5.76. SsSte 20 kinase domain shows $63 \%$ identity with that of Don3, a germinal centre kinase (GCK) from Ustilago maydis. Two exons and one intron are identified within the $2578 \mathrm{bp}$ SsSte20 genomic DNA sequence of S. schenckii. Differential expression of the SsSte20 was demonstrated by real-time RT-PCR. The expression of SsSte20 was much higher in the yeast stage compared with that in the mycelial stage, which indicated that the SsSte20 may be involved in the pathogenesis of the yeast phase of S. schenckii.
\end{abstract}

\section{Introduction}

Sporothrix schenckii (S. schenckii), the etiologic agent of sporotrichosis, is a dimorphic fungus that produces lymphocu-

Correspondence to: Professor Xiaoming Liu or Dr Zhenying Zhang, Department of Dermatology, The First Affiliated Hospital of Dalian Medical University, 222 Zhongshan Road, Dalian, Liaoning 116011, P.R. China

E-mail: liuxiaoming1956@yahoo.cn

E-mail: zhangzhenying7983@yahoo.com.cn

*Contributed equally

Key words: SsSte20, Sporothrix schenckii, gene cloning, differential expression taneous lesions. The mechanisms involved in the morphological transitions of S. schenckii remain unknown, although knowledge on the experimental control of these processes has increased in the past years $(1,2)$. Inhibition of the differentiation of $S$. schenckii mycelial into the parasitic yeast forms may be a noteworthy alternative for the design of antifungal drugs to be used in sporothrichosis. Therefore, understanding the morphological transition process and, consequently, how the morphological transition can be inhibited, may aid in the development of novel strategies to effectively manage this increasingly important clinical problem.

Dimorphic switching requires fungal cells to undergo changes in polarized growth in response to environmental stimuli. Polarity establishment allows the development of a wide variety of cell morphologies and the differentiation of distinct cell types (3). The family of Ste20-related kinases is conserved from yeast to mammals and includes the p21 activated kinases (PAKs) and germinal centre kinases (GCKs). These kinases have been shown to be involved in signaling through mitogen activated protein kinase (MAPK) pathways and in morphogenesis through the regulation of cytokinesis and actin-dependent polarized growth (4). Saccharomyces cerevisiae (S. cerevisiae) Ste20 is required for efficient activity of the pheromone response pathway and an effective Hog1mediated hypertonic stress response. It appears to play a role in the activation of myosin, adhesion of mating partners and vegetative functions relating to budding (5). Homologues of $S$. cerevisiae Ste20 have been described in a number of other fungal species. These include Candida albicans (C.albicans) Ste20 (Q92212), Cryptococcus neoformans (C. neoformans) Ste20 (AF542531.1), Ustilago maydis Ste20 (AF299352.1) and Pneumocystis carinii Ste20 (AF332388). These Ste20 proteins were proved to play important roles in mediating the regulation of various aspects of morphogenesis, conjugation, mating and pathogenicity (6). We previously reported differentially expressed proteins between the mold and early yeast stage of S. schenckii using 2DE (7). The protein spot $\mathrm{J}$ is homologous to the Ste20-like protein kinase from Paracoccidioides brasiliensis and increases in the early yeast form of S. schenckii.

In this study, we described the molecular cloning of the S. schenckii Ste20 homologue, designated SsSte20. We performed necessary function analysis of the SsSte20 gene 
and detected the differential gene expression in dimorphic switch of $S$. schenckii. These will establish the primary foundation of understanding the function of the SsSte20 gene from $S$. schenckii. The cloning and characterization of the SsSte20 gene from $S$. schenckii is reported for the first time.

\section{Materials and methods}

Fungal strain, media and growth conditions. The strain of S. schenckii used, ATCC10268, was maintained at the Research Center for Pathogenic Fungi, Dalian Medical University, China. To obtain a mycelial culture, the ATCC10268 isolate was inoculated on Sabouraud dextrose agar (SDA) medium and incubated at $25^{\circ} \mathrm{C}$. The mycelial colonies thus obtained were inoculated in Sabouraud's fluid medium and cultured with shaking at $100 \mathrm{rpm}$ at $25^{\circ} \mathrm{C}$ for $96 \mathrm{~h}$. To achieve the switch of $S$. schenckii from the mycelial phase to the yeast phase, mycelial colonies were transferred to brain heart infusion (BHI) liquid medium at $37^{\circ} \mathrm{C}$ and shaken at $100 \mathrm{rpm}$ for $96 \mathrm{~h}$. Mycelial and yeast pellets were collected by centrifugation and stored at $-80^{\circ} \mathrm{C}$.

Total RNA, genomic DNA isolation and gene cloning. Approximately $100 \mathrm{mg}$ samples of $S$. schenckii mycelial and yeast were separately pulverized under liquid nitrogen with a mortar and pestle. Total RNA isolation was carried out according to the manufacturer's protocol using the TRIzol Reagent Kit (Invitrogen, Carlsbad, CA, USA) and treated with the RNase-free DNase I kit from Takara to eliminate DNA contamination. Genomic DNA was isolated from yeast phase colonies following the manufacturer's protocol using the InstaGene ${ }^{\mathrm{TM}}$ Matrix kit (Bio-Rad, Hercules, CA, USA). cDNA was synthesized from $500 \mu \mathrm{g}$ of total RNA of ATCC10268 by murine leukemia virus reverse transcriptase (MLV-RT) (Takara, Tokyo, Japan) primed with oligo(dT) following the manufacturer's instructions, and used as template for PCR. Degenerate primers, SsSte20-F1 and SsSte20-R1, were designed based on multiple alignments of the high conserved Ste20 domains of $C$. albicans Ste20 (Q92212), C. neoformans Ste20 (AF542531.1), Ustilago maydis Ste20 (AF299352.1), and Pneumocystis carinii Ste20 (AF332388) amino acid sequences. PCR product of expected size was cloned into pMD18 vector (Takara) and sequenced. The degenerate primers yielded a $302 \mathrm{bp}$ fragment homologous to known Ste20. To obtain the full-length cDNA sequence of the SsSte20 gene, 5'-RACE and 3'-RACE were performed with 5'-Full RACE Kit and 3'-Full RACE Core Set Ver.2.0 kit (Takara) according to the manufacturer's instructions. Nest-PCR was performed. Briefly, five specific primers CTE869 F and CTE869 R of 3'-RACE and R132-1, R132-2 and R132-3 of 5'-RACE were synthesized based on the cDNA sequence obtained by the degenerate primers. PCR products of 5'- and 3'-RACE were both cloned into pMD18 vector (Takara) and sequenced.

To determine the nucleotide sequence of the genomic DNA corresponding to the SsSte20, PCR was performed using the primers SsSte20-P1 and SsSte20-B3 and genomic DNA as template. The PCR products were then sequenced. The sequences of all the primers used in this study are listed in Table I.
Table I. Sequence of primers in this study.

\begin{tabular}{ll}
\hline Primer & \multicolumn{1}{c}{ Sequence (5'-3') } \\
\hline$S s G C K-F 1$ & CAYAARYTNTGGATHGTNATGGA \\
$S s G C K-R 1$ & TGYTGDATNACYTCNGGNGCCATCCA \\
CTE869F & AGAAGCTCCACCGCGATATCAAGGCC \\
CTE869R & CGCCACCATGACCAAGAAGAACACCT \\
R132-1(GSP1) & TGGAGCCTAACAAGACA \\
R132-2(GSP2) & TTCTTGTCGGCGTGGAGGTAG \\
R132-3(GSP3) & CAATGATGGCAATGTAGTCTTCG \\
$P 1$ & ATGGCGAGCAGAGATGACG \\
$B 3$ & CACGGCCTCTTCGTCGAG \\
F & GCAACAGCAACAATTAGC \\
$\mathrm{R}$ & GACACGTTCTCTTCCATG \\
$24 \mathrm{~T}$ & (FAM) CCAACAGCAACTACAACAGCAACAG
\end{tabular}

Degenerate primers designed based on multiple alignments of the high conserved STE20 domains for gene cloning: GCK-F1 and GCK-R1. Primers of 3'-RACE: CTE869 F3, CTE869 F4; 5'-RACE: R132-1, R132-2 and R132-3. To determine the nucleotide sequence of the genomic DNA corresponding to the $S s G C K$ PCR was performed using the primers $P 1$ and $B 3$. Primers and a TaqMan probe of real-time RT-PCR: 8F, 58R and 24T.

Bioinformatic and phylogenetic analysis of SsSte20. Nucleotide sequences and deduced amino acid sequences of the cloned SsSte20 gene were analyzed. The nucleotide sequences were analyzed as previously described by us (8) using Sequencer software (Sequencer, USA) and the BLAST network service of the National Center for Biotechnology Information (NCBI) (http://www.ncbi.nlm.nih.gov/blast). The open reading frame (ORF) was found by the ORF Finder (http://www.ncbi.nlm.nih. gov/gorf/gorf.html). For the exact localization of the exon/intron boundaries, the mRNA-to-genomic alignment program Spidey (http://www.ncbi.nlm.nih.gov/IEB/Research/Ostell/Spidey/ index.html) was used. The deduced amino acid sequence was analyzed with the Expert Protein Analysis System (http://www. expasy.org/) and the protein domain features of SsSte20 were determined by using Simple Modular Architecture Research Tool (http://hits.isb-sib.ch/cgi-bin/PFSCAN). Isoelectric point and molecular weight prediction were carried out at (http:// cn.expasy.org/tools/pi_tool.html). Multiple alignments of SsSte20 were performed with the ClustalW Multiple Alignment Program (http://www.ebi.ac.uk/clustalw/).

Differential expression of SsSte20 in two stages during dimorphic switch. The expression of SsSte20 transcript in different stages (mycelial, yeast) was measured by real-time RT-PCR. Primers and a TaqMan probe for target genes were designed with Primer Select in DNASTAR software (Lasergene) and are listed in Table I (24T, 8F and 58R). Fifty nanograms of total RNA were assayed from two stages of $S$. schenckii in triplicate using the PrimeScript RT-PCR Kit (Takara). The minus-reverse transcriptase control was also performed in triplicate. The amplification conditions were optimized for the ABI-PRISM-7500 instrument (Applied Biosystems). The cycling conditions using TaqMan probe detection were $95^{\circ} \mathrm{C}$ for $2 \mathrm{~min}$ followed by 40 cycles at $95^{\circ} \mathrm{C}$ for $10 \mathrm{sec}, 61^{\circ} \mathrm{C}$ for $10 \mathrm{sec}, 72^{\circ} \mathrm{C}$ for $40 \mathrm{sec}$. $18 \mathrm{srDNA}$ was selected as the endog- 
Table II. Relative abundance of differential expression gene as determined by real-time RT/PCR $(\mathrm{mean} \pm \mathrm{SD})(\mathrm{P}<0.01)$.

\begin{tabular}{lcccccc}
\hline cDNA name & Phase & Target $C_{\mathrm{T}}$ & 18 srDNA C & $\Delta \mathrm{C}_{\mathrm{T}}$ & \multicolumn{2}{c}{$2^{-\Delta \Delta \mathrm{C}_{\mathrm{T}}}$} \\
\hline SsSte20 & Mycelial & $22.48 \pm 0.92$ & $20.66 \pm 0.25$ & $1.81 \pm 1.13$ & $0 \pm 1.13$ \\
& Yeast & $19.99 \pm 0.23$ & $22.09 \pm 0.60$ & $-2.11 \pm 0.79$ & $-3.92 \pm 0.79$ \\
\hline
\end{tabular}

$\Delta \mathrm{C}_{\mathrm{T}}$, target transcript $\mathrm{C}_{\mathrm{T}}-18 \mathrm{srDNA} \mathrm{C}_{\mathrm{T}}$ normalization of $\mathrm{C}_{\mathrm{T}}$ for target gene relative to $18 \mathrm{srDNA} \mathrm{C}_{\mathrm{T}}$. Statistical analysis of normalized expression levels between mycelial form and yeast form. Each of the target genes differs significantly (U-test, $\mathrm{P}<0.05$ ). $\Delta \Delta \mathrm{C}_{\mathrm{T}}$, mean yeast $\Delta \mathrm{C}_{\mathrm{T}}$, mean mycelial $\Delta \mathrm{C}_{\mathrm{T}}$. The mean value for the mycelial $\Delta \mathrm{C}_{\mathrm{T}}$ was used as a calibrator to set the baseline for comparing mean differences in the $\Delta \mathrm{C}_{\mathrm{T}}$ values of yeast form. $2^{-\Delta \Delta \mathrm{C}_{\mathrm{T}}}$, normalized target amount relative to the mycelial form.

enous control. Relative quantification of target gene expression was evaluated using the comparative cycle threshold $\left(\mathrm{C}_{\mathrm{T}}\right)$ method as previously described by Livak and Schmittgen (9). The $\Delta \mathrm{C}_{\mathrm{T}}$ value was determined by subtracting the target $\mathrm{C}_{\mathrm{T}}$ of each sample from its respective $18 \mathrm{srDNA} \mathrm{C}_{\mathrm{T}}$ value. Calculation of $\Delta \Delta \mathrm{C}_{\mathrm{T}}$ involved using the mycelial sample $\Delta \mathrm{C}_{\mathrm{T}}$ value as an arbitrary constant to subtract from yeast sample $\Delta C_{T}$ values. Differences in expression of target genes were determined by $2^{-\Delta \Delta \mathrm{C}_{\mathrm{T}}}$. Data are expressed as arithmetic means \pm SD unless otherwise indicated. Comparison between mycelial and yeast samples was performed using the Student's t-test. Differences with a P-value of $<0.05$ were considered statistically significant.

\section{Results}

Cloning and genomic structure of SsSte20. A full-length SsSte20 cDNA (2,846 bp) including an ORF of $2505 \mathrm{bp}$, encoding 835 amino residues, was flanked by a $41 \mathrm{bp}$ 5'-untranslated region (5'-UTR) and a 297 bp 3'-UTR. The SsSte 20 genomic DNA is 2578 bp in length. The aligned results revealed that there is one intron between the sequences of the genomic DNA and the cDNA. Its 5' and 3' ends conformed to the basic consensus, GT/AG, for the eukaryotic splice donor and acceptor site. Based on the sequence of cDNA, the molecular weight of the predicted amino acid is $\sim 91.31 \mathrm{kDa}$, the theoretical pI is 5.76 .

Motif searches and sequence comparison showed that SsSte20 consists of a 251-amino acid N-terminal kinase domain (residues 21-271) (Fig. 1). In the N-terminal extremity of the catalytic domain there is a glycine-rich stretch of residues in the vicinity of a lysine residue $(\mathrm{K})$, which has been proved to be involved in ATP binding. In the central part of the catalytic domain there is a conserved aspartic acid residue $(\mathrm{N})$ which is important for the catalytic activity of the enzyme (Fig. 2). One proline/glutamic acid/serine/threonine (PEST) motif was searched using Epestfind in the C-terminal domain of SsSte20 (residues 514-529) (Fig. 1). Database searches revealed that SsSte20 is a member of the Ste20 family of protein kinases. SsSte20 displays high homology throughout the kinase domain and contains the amino acid sequence GTPYFWMAPEV in the kinase domain (Fig. 2). This sequence motif, known as the Ste20 signature, is characteristic of the Ste20 family of protein kinases and is critical for the kinase activity of Ste20 (10).

Homology and phylogenetic analysis of SsSte20. To clarify the relationship between SsSte20 and other Ste20-related kinases, we calculated multiple sequence alignments of these sequences.
The derived evolutionary tree is split into two main branches, one formed by some PAKs, the other one by GCKs including SsSte20 sequence (Fig. 3). Further alignment clearly showed that the SsSte20 kinase domain is highly homologous to that of other GCKs, such as Don3 (Ustilago maydis, AF463451.1), Ppk11 (Schizosaccharomyces pombe, NM_001019946.1), Sid1 (Schizosaccharomyces pombe, NM_001018997.1), Nak1 (Schizosaccharomyces pombe NM_001021931.1) and polaritydefective 6 (Neurospora crassa, DQ336953.1). These proteins share a similar structure and have $63,56,54,47$ and $38 \%$ amino acid similarities in the catalytic domains, respectively. These proteins have a highly conserved catalytic domain in common but differ in their C-terminal regulatory domain, which does not contain any identifiable sequence motifs.

Expression of SsSte20 in two stages of S. schenckii. The mRNA expression of SsSte20 in different stages was analyzed by real-time RT-PCR normalized against $18 \mathrm{srDNA}$ levels. Expression was determined as fold increased $2^{-\Delta \Delta \mathrm{C}_{\mathrm{T}}}$ levels relative to the stage with lowest expression (mycelial) set to 1 . The SsSte20 gene was expressed in two stages of S. schenckii, with higher mRNA levels observed in yeast (15.14-fold). There were significant differences between the mycelial and the yeast form (Table II).

Accession number. The full length of cDNA sequence and the genomic DNA sequence of the SsSte20 gene were submitted to the GenBank database under the accession numbers JX312329 and JX857536, respectively.

\section{Discussion}

The Ste20-like protein kinase family can be divided into two subfamilies based on their domain structure and regulation. The PAK/Ste20 subfamily contains a C-terminal catalytic domain and an N-terminal binding site for the small G proteins $\mathrm{Cdc} 42$ and Rac1, which are considered to be key regulatory molecules linking surface receptors to the organization of the actin cytoskeleton $(11,12)$. By contrast, the kinases of the second subfamily, the GCK subfamily, have an N-terminal kinase domain and a C-terminal regulatory domain that does not have a recognizable GTPase binding site (13). Furthermore, GCKs are subdivided into two broad groups based on their structure. Group I GCKs have a highly conserved C-terminal domain containing at least two PEST motifs, two polyproline Src homology-3 (SH3) domains and an additional highly conserved region of approximately 350 


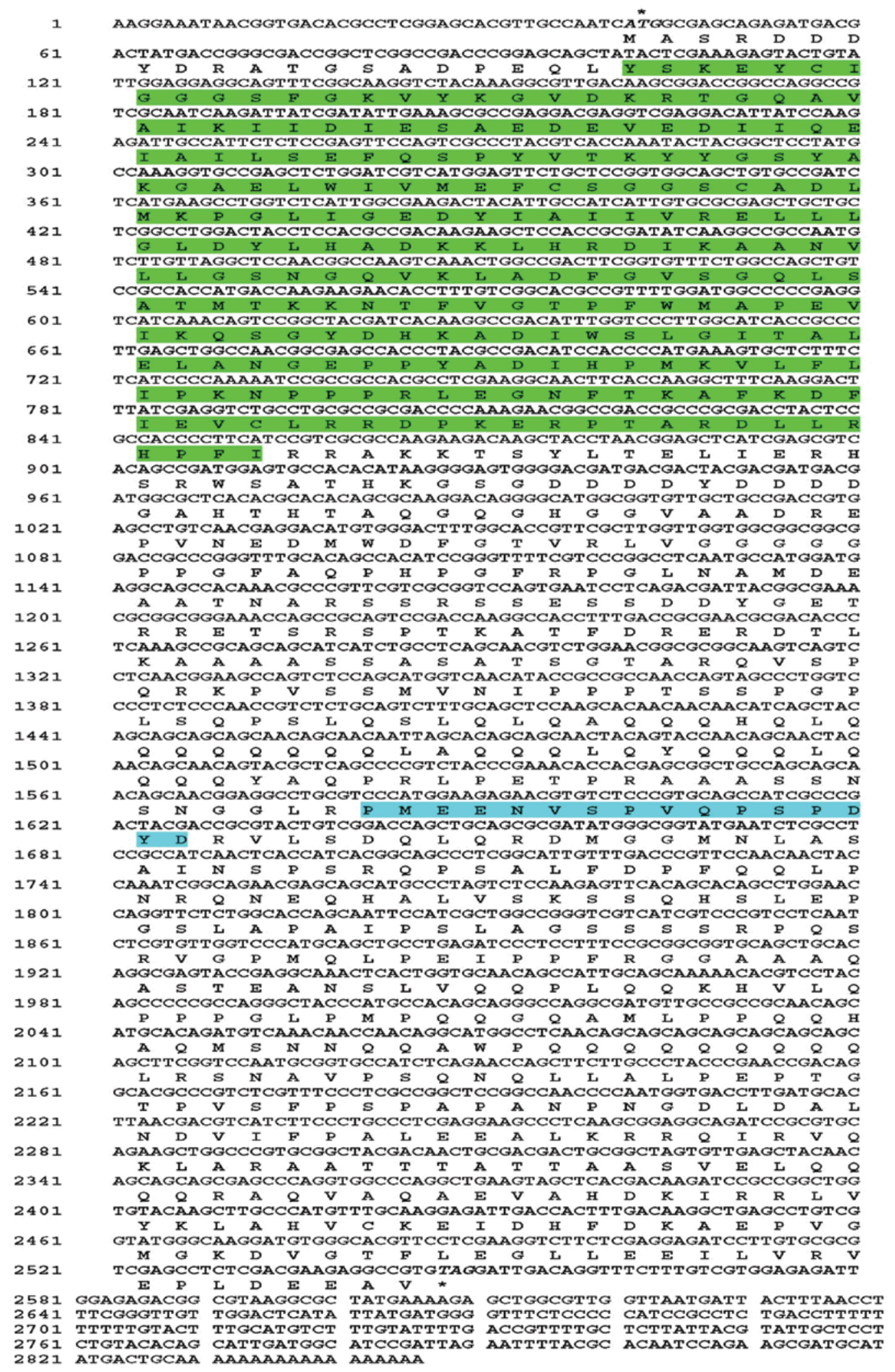

Figure 1. Nucleotide sequence of the SsSte20 and the predicted amino acid sequence of the SsSte20 product. The cDNA sequence of SsSte20 is listed in the top lines. Deduced amino acid sequence of ORF is shown by the single-letter amino acid codes. Start codon (ATG) and stop codon (TAG) are indicated by bold and *. S_TKc(Serine/Threonine protein kinases, catalytic domain) domain is shaded in green. PEST MOTIF is shaded in blue.

amino acids. Group II GCKs share similarity to the catalytic domain of group I; however, the C-terminal domain differs significantly. In present study, SsSte20 was labelled as a GCK based on catalytic domain located in the $\mathrm{N}$-terminal site, which was also confirmed by phylogenetic relationship analysis. We further identified SsSte20 as a group II GCK for its one PEST motif, no SH3 domain and no conserved sequence in C-terminal domain. 


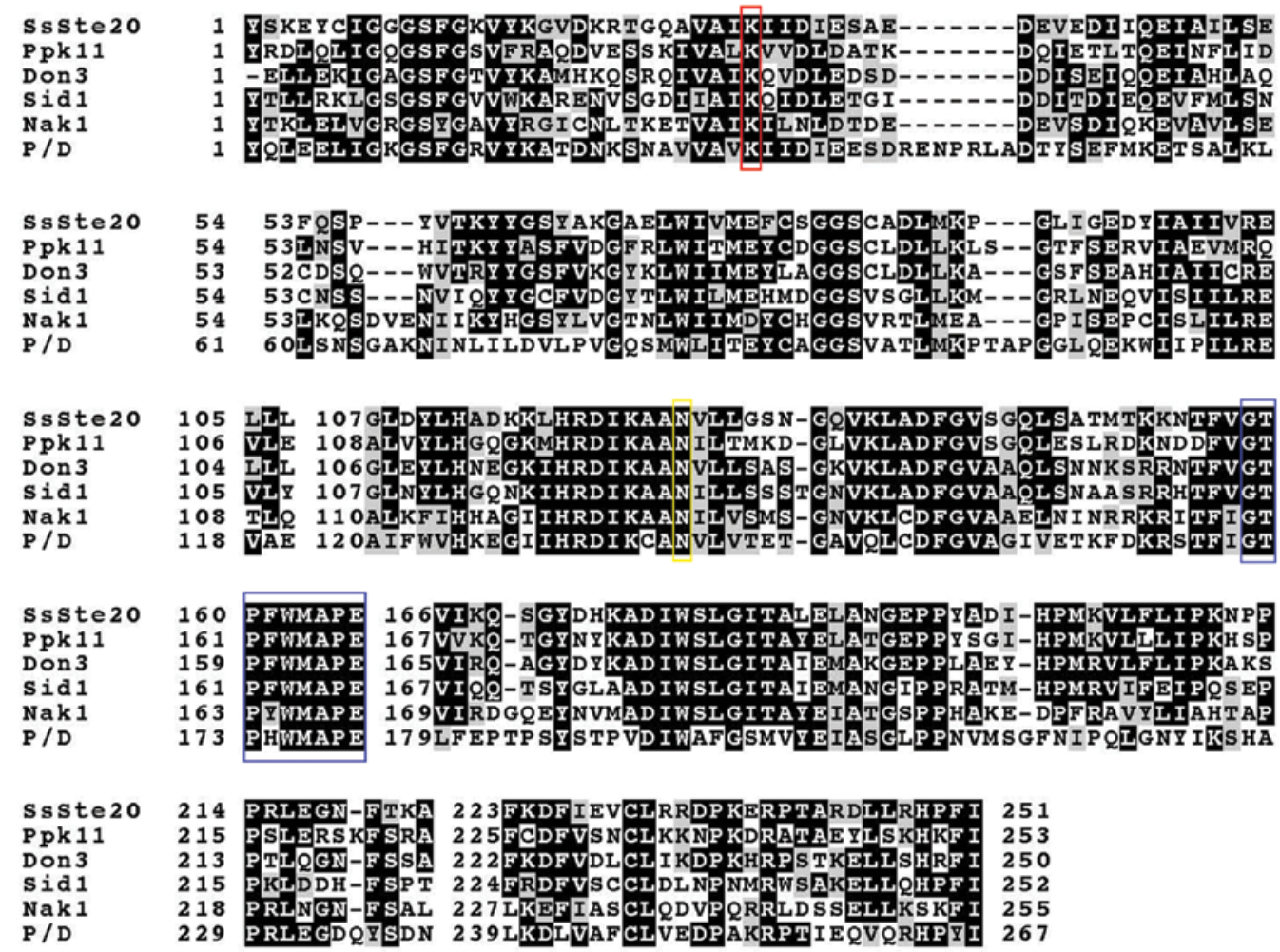

Figure 2. The alignment of the predicted amino acid sequence of the SsSte20 kinase domain with the corresponding domains of Don3, Ppk11, polaritydefective 6 (P/D), Nak1 and Sid1 is shown. Sequences were aligned using the ClustalW and shaded by BOXSHADE. Shade residues indicate $\geq 75 \%$ homology (black) or $\geq 50 \%$ homology (gray). The conserved Lysine (K) and aspartic acid residue (N) are indicated by a red and yellow box, respectively. The PAK/Ste20 signature sequence is indicated by a blue box.

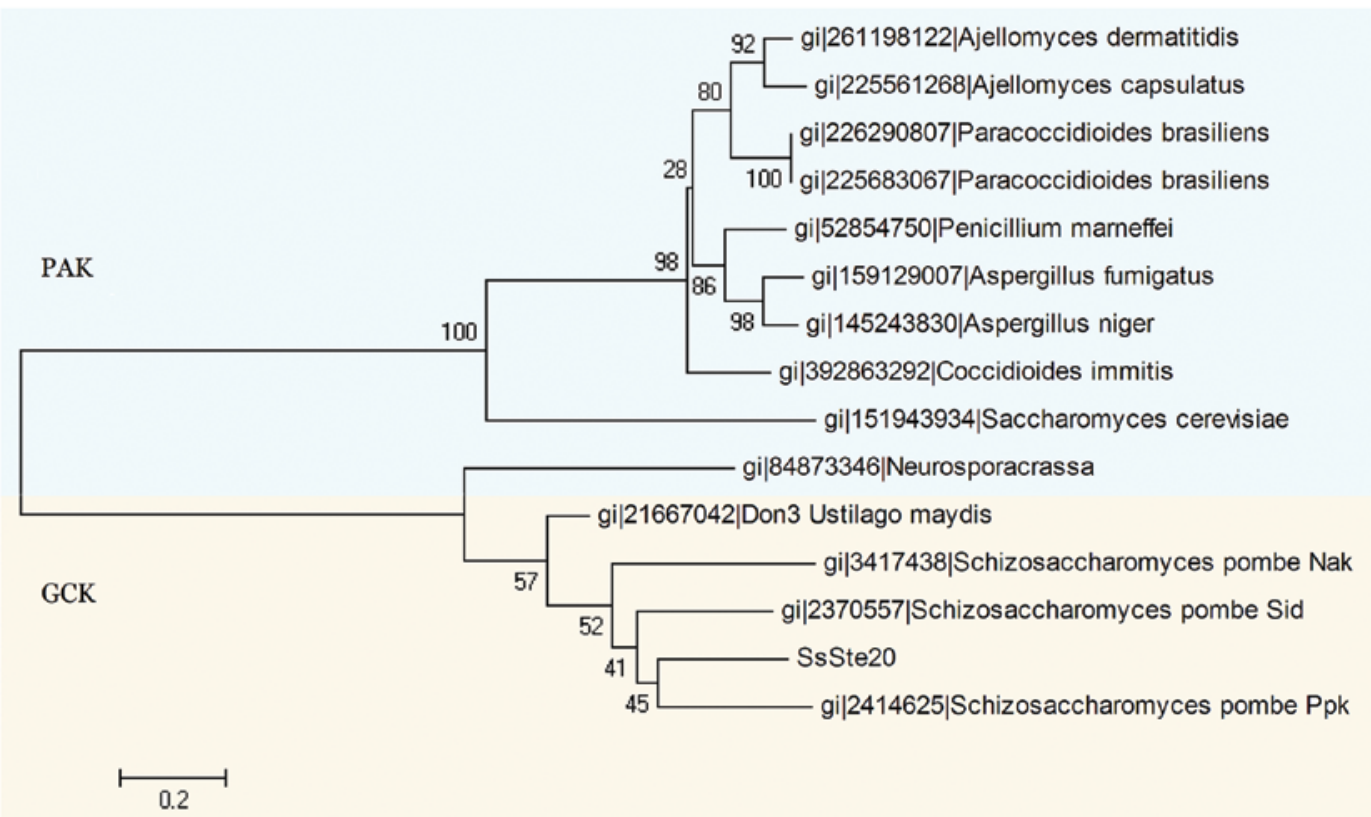

Figure 3. Phylogenetic relationship among S/TPK homologues. An NJ tree was generated by MEGA version 5.05 with bootstrap analysis based on 500 replications. Percentage bootstrap values are shown at branch points. Scale bar indicates the number of substitutions per site.

Ste20 ortholog was proved to be involved in both the morphogenesis and the pathogenesis of consequence of an inability to grow in the pathogenic form. In C. albicans, filamentous growth allows adherence and penetration of tissues during infection. The cst20 (Ste20) mutants are unable to form hyphal filaments in vitro and consequently show reduced kidney colonization and virulence in a mouse model for systemic candidiasis (14). While in Penicillium marneffei 
(P. marneffei), infection occurs by the inhalation of conidia that are phagocytosed by host pulmonary alveolar macrophages where they germinate into yeast cells and divide by fission. PakA (Ste20) plays an essential role in conidial germination of P.marneffei at the host temperature of $37^{\circ} \mathrm{C}$ such that $\triangle$ PakA mutant conidia fail to germinate during macrophage infection (15). Although GCKs currently have no described role in virulence, this may be due to a lack of characterization rather than a lack of involvement. The Kic1 (GCK) mutants in $C$. albicans and $C$.neoformans probably have virulence defects as the $C$.albicans Kic1 mutant is unable to grow filamentously and the $C$. neoformans mutant is unable to grow at $37^{\circ} \mathrm{C}$, the temperature of the human body, both of which are requirements for virulence in these organisms $(16,17)$. In S. schenckii, the germination of conidia is a key pathogenicity determinant since conidia are the infectious propagules. Does SsSte20, a GCK, have the same function in the virulence of $S$. schenckii as Ste20 ortholog in other fungi? The mRNA expression of SsSte20 in yeast cells of both ATCC10268 and a clinical S. schenckii isolated from a patient with fixed sporotrichosis (data not shown) were higher than the mycelial ones, which suggested that SsSte20 may be involved in the morphogenesis or pathogenesis of S. schenckii.

What is the environmental signal that SsSte20 responds to to participate in morphogenesis and pathogenesis? In P. marneffei, PakB (Ste20) is essential for the formation of yeast cells within macrophages and the $\triangle \mathrm{PakB}$ mutant grows as septate hyphae in vivo. PakB is not expressed at $37^{\circ} \mathrm{C}$ during in vitro yeast growth but expression is induced during macrophage infection (18). This suggests that unlike PakA, which responds to temperature, PakB responds to host cell inductive signals. In the present study, the mycelial cells of S. schenckii switched to yeast cells with obvious increased expression of SsSte20 when they were incubated in BHI liquid medium at $37^{\circ} \mathrm{C}$ in vitro, which suggests SsSte20, similar to PakA, can detect the change of temperature and nutrient deprivation in the environment. Whether SsSte20 also responds to other host signals remains to be elucidated. It is most likely that there are other proteins that fungal cells come into contact with in the early stages of infection. We are carrying out further experiments on generating SsSte20-mutants to study its detailed functions in this critical fungal pathogen.

\section{Acknowledgements}

This study was partly supported by a grant from the National Natural Science Foundation of China (Grant no. 81000069).

\section{References}

1. Valle-Aviles L, Valentin-Berrios S, Gonzalez-Mendez RR and Rodriguez-Del Valle N: Functional, genetic and bioinformatic characterization of a calcium/calmodulin kinase gene in Sporothrix schenckii. BMC Microbiol 7: 107, 2007.
2. Valentín-Berríos S, González-Velázquez W, Pérez-Sánchez L, et al: Cytosolic phospholipase $\mathrm{A}_{2}$ : a member of the signalling pathway of a new G protein $\alpha$ subunit in Sporothrix schenckii. BMC Microbiol 9: 100, 2009.

3. Boyce KJ, Hynes MJ and Andrianopoulos A: The Ras and Rho GTPases genetically interact to co-ordinately regulate cell polarity during development in Penicillium marneffei. Mol Microbiol 55: 1487-1501, 2005.

4. Boyce KJ and Andrianopoulos A: Ste20-related kinases: effectors of signaling and morphogenesis in fungi. Trends Microbiol 19: 400-410, 2011

5. Calcagno AM,Bignell E, Rogers TR, Canedo M, MuhlschlegelFA and Haynes K: Candida glabrata Ste20 is involved in maintaining cell wall integrity and adaptation to hypertonic stress, and is required for wild-type levels of virulence. Yeast 21: 557-568, 2004

6. Hazan I and Liu H: Regulation of morphogenesis by conserved developmental pathways in pathogenic fungi. In: Pathogenic Fungi: Structural Biology and Taxonomy. San-Blas G and Calderone RA (eds). Caister Academic Press, Norfolk, UK, pp221-229, 2004.

7. Zhang ZY, Hou BB, Xin Y and Liu XM: Protein profiling of the dimorphic pathogenic fungus, Sporothrix schenckii. Mycopathologia 173: 1-11, 2012.

8. Hou B, Zhang Z, Zheng F and Liu X: Molecular cloning, characterization and differential expression of DRK1 in Sporothrix schenckii. Int J Mol Med 31: 99-104, 2013.

9. Livak KJ and Schmittgen TD: Analysis of relative gene expression data using real-time quantitative PCR and the 2(-Delta Delta C(T)) method. Methods 25: 402-408, 2001.

10. Knighton DR, Zheng JH, Ten Eyck LF, Xuong NH, Taylor SS and Sowadski JM: Crystal structure of the catalytic subunit of cyclic adenosine monophosphate-dependent protein kinase. Science 253: 407-414, 1991.

11. Wu C, Whiteway M, Thomas DY and Leberer E: Molecular characterization of Ste20p, a potential mitogen-activated protein or extracellular signal-regulated kinase kinase (MEK) kinase kinase from Saccharomyces cerevisiae. J Biol Chem 270: 15984-15992, 1995.

12. Herskowitz A, Choi S, Ansari AA and Wesselingh S: Cytokine mRNA expression in postischemic/reperfused myocardium. Am J Pathol 146: 419-428, 1995.

13. Hall A: Rho GTPases and the actin cytoskeleton. Science 279: 509-514, 1998.

14. Leberer E, Harcus D, Clark KL, Dignard D, Ziegelbauer K, et al: Signal transduction through homologs of the Ste20p and Ste7p protein kinases can trigger hyphal formation in the pathogenic fungus Candida albicans. Proc Natl Acad Sci USA 93: 13217$13222,1996$.

15. Boyce KJ and Andrianopoulos A: A p21-activated kinase is required for conidial germination in Penicillium marneffei. PLoS Pathog 3: e162, 2007.

16. Walton FG, Heitman, J Boone AC and Editor M: Conserved elements of the RAM signaling pathway establish cell polarity in the basidiomycete Cryptococcus neoformans in a divergent fashion from other fungi. Mol Biol Cell 17: 3768-3780, 2006.

17. Song Y, Cheon SA, Lee KE, Lee S, Lee B, et al: Role of the RAM network in cell polarity and hyphal morphogenesis in Candida albicans. Mol Biol Cell 19: 5456-5477, 2008.

18. Boyce KJ, Schreider L and Andrianopoulos A: In vivo yeast cell morphogenesis is regulated by a p21-activated kinase in the human pathogen Penicillium marneffei. PLoS Pathog 5: e1000678, 2009. 